\title{
Special Issue: Advances in Multiphase-Flow Modelling and Measurement
}

\author{
Erick Franklin $^{1}$ (D) Gijsbert Ooms ${ }^{2} \cdot$ Martin Sommerfeld $^{3}$
}

Published online: 16 August 2020

(c) Springer Nature B.V. 2020

\section{Preface}

This Special Issue contains 18 papers presented at the 10th International Conference on Multiphase Flow-ICMF2019. The papers are extended versions of a larger subset of conference contributions that were chosen from 130 manuscripts accepted by the ICMF2019 Scientific Committee for presentation at the conference. The selected manuscripts were then subjected to the usual, stringent peer-review process, by three referees, as done with any other manuscripts submitted to FTaC. Thus, the papers contained in this Issue are those which successfully passed a three-stage review and selection process.

The papers included herein report progress in a broad range of experimental and numerical studies, including advanced techniques such SPH, deep machine learning, PIV and LDA of complex multi-phase flows containing particles, droplets and bubbles. Specifically, the papers document studies on numerical techniques for droplet-particle and particle-particle collisions within a carrier flow and two-phase compressible flows within the low-Mach-number regime; deep learning techniques in conjunction with numerical analysis of two-phase flows; experimental and numerical investigations of two-phase jets, sprays, atomizers and drop-pool impact; experiments on three-phase flows, bubbly flows around a tube bundle and two-phase flows in micro-channels; modeling of bubble breakage and coalescence considering bubble-induced turbulence and reduced order schemes for two-phase flows; numerical simulations of microbubbles in turbulent flows and turbulent particle-laden flows; and an experimental study of interfacial dynamics at high pressure. This reflects the wide range of research topics studied in the field of multiphase flows. Therefore, this FTaC Issue may be claimed to represent the state of the art in the field of multiphase flows at the time of publication, and the editors hope that readers will appreciate its contribution and value its content.

Erick Franklin, University of Campinas, Brazil, Guest Editor

Gijsbert Ooms, Delft University of Technology, The Netherlands, Guest Editor

Martin Sommerfeld, Otto-von-Guericke University Magdeburg, Germany, Guest Editor

Erick Franklin

franklin@fem.unicamp.br

1 University of Campinas, Campinas, Brazil

2 Delft University of Technology, Delft, The Netherlands

3 Otto-von-Guericke University Magdeburg, Magdeburg, Germany 